\title{
Essais d'infestation du Lérot par Strongyloides ratti (Nématodes Phasmidiens.)
}

\author{
par E. ROMAN, J. MICHEL-BRUN et M.-A. POTHIER \\ Laboratoire de Parasitologie ( $P^{r}$ J. Coudert), Faculté de Médecine et de Pharmacie F 69 - Lyon
}

\section{Résumé}

\begin{abstract}
Alors que tous les hôtes connus de Strongyloides ratti appartiennent au groupe des rats vrais, quelques infestations légères, et même une réinfestation, par ce Nématode ont pu être obtenues expérimentalement chez un Myoxidae, le lérot, Eliomys quercinus. Cette relative résistance à la pénétration de l'helminthe doit, compte-tenu des mœurs arboricoles, expliquer pourquoi, avec les examens de routine, aucun individu de ce dernier Rongeur n'a été trouvé spontanément parasitr par $S$. ratti dans des localités des environs de Lyon, où divers Muridae des campagnes le sont souvent.
\end{abstract}

\section{Summary}

Though all the known hosts of Strongyloides ratti belong to the group of true rats, some slight infections - and even reinfection - with this Nematode may be experimentally obtained in a Myoxidae, the Garden dormouse Eliomys quercinus.

The relative resistance of this host to the penetration of the Helminth may be attributed to its tree-dwelling habits, as, in localities close to Lyon, several kinds of fields Muridae are often infested, but routine tests never found any Eliomys quercinus bearing spontaneously $S$, ratti. 
Dans le présent mémoire sont exposées nos tentatives d'infestation de lérots, Eliomys quercinus (L.), avec deux souches de Strongyloides ratti Sandground, que nous conservons par passages réguliers sur Rattus norvegicus (Erxel.) albinos; l'une, «Villars », entretenue depuis 1963 provient d'un mulot, Apodemus sylvaticus (L.) (voir E. Roman, 1964, 1966), l'autre, "St-André», a été isolée d'un campagnol, capturé sur la commune de Limonest (Rhône), que J. Batteta, du Muséum de Lyon, à qui nous adressons nos remerciements, rapporte à Clethrionomys glareolus (Schreber).

Ces essais ont été réalisés par balnéation, suivant les indications de A. ArrezaGuzman (1937). Chez les rats de laboratoire, au moins 1/50 des larves filariformes ainsi administrées devient adulte dans l'intestin grêle (E. Roman, 1956); elles peuvent alors être mises en évidence dans la paroi de cet organe, à l'autopsie des animaux parasités, en examinant avec un faible grossissement le produit du raclage de sa muqueuse comprimé entre deux lames.

Chez tous les Rongeurs que nous observons, notamment chez ceux servant à nos expériences, nous dépistons $S$. ratti par la recherche directe des œufs et des larves rhabditoïdes dans environ 1 centig. de déjections ou du contenu de l'intestin terminal, dilué dans une goutte d'eau physiologique. En cas de résultat négatif, nous avons effectué occasionnellement l'enrichissement de ces productions par une méthode préconisée par l'un de nous (1957), pour les selles riches en résidus cellulosiques. Des coprocultures, suivant la méthode simplifiée attribuée à E. Brumpt par M. Langeron (1934), ont été, dans les mêmes circonstances, plus souvent réalisées ; la centrifugation de leur phase liquide nous a souvent permis de mettre en évidence des larves filariformes de $S$. ratti dans les faibles infestations.

Il était intéressant de connaître la réceptivité à Strongyloides ratti d'un représentant de la famille des Myoxidae, parce que, jusqu'à récemment, ce Nématode n'était reconnu parasite que chez des Muridae du groupe des rats vrais; il peut, cependant, infester Mesocricetus auratus Waterh. et Meriones tristrami Thomas (G. Wertheim, 1959) (1), Rongeurs de cette superfamille, assez éloignés zoologiquement des espèces du genre Rattus, hôtes les plus favorables de cet Helminthe.

Par ailleurs, en Europe occidentale, la question de la réceptivité de Eliomys quercinus à $S$. ratti présente un intérêt écologique certain. Dans un mémoire sur les Acanthocéphales et les Nématodes chez les rats de la région lyonnaise, l'un de nous en 1951 ne signalait aucun représentant de ces groupes zoologiques chez sept lérots autopsiés les années précédentes, après examen direct du contenu intestinal, tandis que $S$. ratti était constaté, en opérant de même, chez des rats des champs de trois autres espèces, capturés dans les mêmes localités du Mont-d'Or lyonnais, Apodemus sylvaticus, Microtus arvalis (Pall.) et Clethrionomys glareolus. Depuis 1951, 63 rats des champs ont été disséqués ; $S$. ratti n'a pas été décelé à l'examen direct du contenu intestinal, chez 21 lérots, provenant, l'un des Monts du Lyonnais, un peu plus de la moitié de St-Didier

(1) Un Meriones shawii Rozet adulte, d'un élevage parvenu de Tunisie à 1'Institut Pasteur de Lyon, sur l'initiative du regretté Paul Durand, a été infesté le 16 juin 1966 avec environ 9.400 larves de $S$. ratti, souche Villars; bien qu'aucun œuf ou larve n'ait été décelé les 22 et 23 juin dans ses crottes, il a été trouvé trois femelles parthénogénétiques de ce Nématode dans la première moitié de son intestin grêle. 
au Mont-d'Or, les autres de différents points des Dombes; parmi les Rongeurs d'autres espèces, sur 11 capturés à Villars-les-Dombes (Ain), le seul Microtus agrestis (L.) et 4 Apodemus sylvaticus ont été reconnus porteurs de Strongyloides par l'examen précédent ou par la coproculture de leurs résidus digestifs.

Notre expérimentation a porté sur 5 lérots adultes, qu'à l'exception d'un seul, le $\mathrm{n}^{\circ} 2$, nous devons à l'amitié de J. Bussy, qui les a capturés en Dombes.

LÉrot 1. - Infesté le 6 juin 1967 avec environ 10.000 larves filariformes de Strongyloides ratti, provenant de coprocultures de crottes de rats servant à l'entretien de la souche Villars.

12. VI. - Examen direct des crottes : un œuf, 2 larves rhabditoïdes de $S$. ratti.

16. VI. - Même examen : 24 œufs, 6 larves rhabditoïdes du même Nématode.

A l'autopsie, pratiquée à cette dernière date, il est trouvé dans l'intestin (il n'y a pas de cæcum chez le lérot) 10 femelles parthénogénétiques de $S$. ratti, 6 dans Ie $1^{\text {er }}$ quart, 3 dans le $2^{\circ}$, une dans le $3^{\circ}$, aucune dans le dernier.

Le 17 juin, un rat albinos a été infesté avec environ 4.300 larves filariformes de $S$. ratti, développées dans les phases liquides des coprocultures des crottes de ce lérot des 13 et 14 de ce mois; six jours après, 24 œufs et 53 larves rhabditoïdes de Strongyloides ont été vus à l'examen direct de ses déjections. Au moment de son autopsie, le $1^{\text {er }}$ août, ce rat était déparasité ; il n'a pas été, en effet, trouvé de femelles parthénogénétiques de ces Nématodes dans son intestin grêle.

LÉrot 2. - Animal capturé très jeune, en captivité depuis 2 ans.

Infesté le 24 mars 1969 avec environ 2.500 larves filariformes de $S$. ratti de souche Villars.

Examens directs des crottes négatifs les 26, 27, 28, 29 et 31 mars, également le $1^{\text {er }}$ et le 2 avril.

5. IV. - 2 larves filariformes vivantes de $S$. ratti dans les phases liquides de chacune des coprocultures effectuées le 31 mars et le $1^{\text {er }}$ avril.

Aucune larve de Nématodes n'est constatée, de même dans celles mises en train les 22,23 et 25 avril.

25. IV. - L'animal est réinfesté avec environ 10.000 larves filariformes de $S$. ratti de même provenance que précédemment.

Il n'est constaté aucune forme évolutive de Strongyloides à l'examen des crottes des 2 et 3 mai.

Parmi les coprocultures effectuées à partir de cette dernière date, celles des 3 et 6 mai montrent respectivement dans la phase liquide 2 et une larve filariforme de $S$. ratti; celles des $11,12,14$ et 16 du même mois sont négatives.

Le 3 juin, l'animal est une deuxième fois réinfesté avec environ 10.000 larves filariformes de même provenance.

Il n'est pas à nouveau constaté de stades évolutifs de Strongyloides à l'examen direct ni, après enrichissement, dans les crottes émises 7,8 et 9 jours plus tard.

Dans les phases liquides de coprocultures effectuées les 10,11, 12, 13, 14, 16 et 17 juin, il n'est observé aucune larve de Nématodes. 
A l'autopsie, le 20 de ce mois, il n'est pas constaté dans l'intestin de femelles parthénogénétiques de Strongyloides.

LÉRot 3. - L'animal est infesté le 5 février 1970 avec environ 4.700 larves filariformes provenant d'un rat hébergeant des $S$. ratti de la souche St-André.

Examens directs des crottes négatifs les 7, 9, 10, 11 et 12 février.

Pas de larves filariformes de $S$. ratti dans les phases liquides des coprocultures des crottes des 12, 13,14, 16 et 17 février.

A l'autopsie, le 17 février, il n'est vu aucune femelle parthénogénétique de Strongyloides dans l'intestin.

LÉRot 4. - Infesté le 17 février 1970 avec environ 11.700 larves filariformes de $S$. ratti de même provenance que dans le cas du lérot 3 .

Pas de stades évolutifs de Strongyloides à l'examen direct des crottes des 18, 19, $20,21,23,24$ et 26 février, mais présence de 2 larves rhabditoïdes de $S$. ratti dans celles du 25.

Dans les phases liquides des coprocultures des crottes des 24, 25, 26 et 27 février. il est constaté respectivement une, 4,3 et 2 larves filariformes de $S$. ratti.

A l'autopsie, le 27 février, il est trouvé une femelle parthénogénétique de Strongyloides tout à fait au début de l'intestin.

LÉROT 5. - Infesté le 26 février 1970 avec environ 13.000 larves filariformes de S. ratti de souche Villars d'une coproculture de crottes d'un rat.

Pas de stades évolutifs de $S$. ratti à l'examen direct des crottes du 27 et du 28 février, ainsi que dans celles des $2,3,4,5,6$ et 7 mars.

Pas de larves filariformes de Strongyloides dans les phases liquides des coprocultures des crottes des 5, 6, 7 et 9 mars.

A l'autopsie, le 9 mars, il n'est trouvé dans l'intestin aucune femelle parthénogénétique de $S$. ratti.

En somme, 3 des 5 lérots, que nous avons soumis à la pénétration de larves filariformes de Strongyloides ratti, ont été reconnus parasités par ce Nématode; l'un, le $\mathrm{n}^{\circ} 1$, a présenté une infestation relativement forte $\left(1 / 1.000^{\circ}\right.$ des larves administrées se sont développées dans son intestin), mais cependant 10 fois plus faible que celle d'un rat albinos éprouvé de la même manière ; chez les lérots 2 et 4 , très peu de larves ont pu se développer, puisque, sauf une fois, tous les examens directs des crottes ont été négatifs et que le parasitisme n'a été révélé qu'à la coproculture ; chez ce dernier, autopsié peu après la maturité sexuelle des femelles parthénogénétiques, un seul de ces Nématodes (moins $1 / 10.000^{\circ}$ des larves administrées) a été trouvé dans son duodénum. En conséquence, si le lérot peut être réceptif à $S$. ratti, il représente pour lui un hôte peu favorable. Nous comparerions volontiers cette susceptibilité à celle de Mus musculus L. albinos, telle qu'elle a été observée par S. Brackett et A. Bliznick (1949), puis par l'un de nous (1956). 
Il est peu probable que nos succès, et aussi nos échecs, soient en rapport avec la souche de Strongyloides utilisée, puisque des résultats positifs et négatifs ont été obtenus avec l'une et l'autre. Il est plus vraisemblable que la diversité de nos résultats tient à des différences individuelles de susceptibilité au Nématode de nos animaux; le lérot 1 était certainement beaucoup plus réceptif à ce parasitisme que d'autres.

Il était intéressant d'infester une deuxième fois un lérot: l'expérience a en effet montré que, chez les rats de laboratoire, une seconde pénétration des larves filariformes de $S$. ratti est en règle générale possible, mais qu'il se développe, la deuxième fois, une moindre proportion de ces stades jeunes que dans la première (A. J. Sheldon, 1937, E. Roman, 1959). L'essai $\mathrm{n}^{\circ} 2$ montre que, chez un hôte peu réceptif, débarrassé de ses parasites de première invasion, ce qui chez notre animal s'est réalisé moins de 22 jours après leur introduction, il y a possibilité d'une $2^{\circ}$ infestation, qui, dans notre cas, a été également de peu de durée. Par contre, la résistance du lérot à une $3^{\circ}$ pénétration de $S$. ratti apparaît totale. D'après ces données, une infestation spontanée de notre $\mathrm{n}^{\circ} 1$, dont les crottes n'ont pas été examinées dès sa mise en observation, ne peut être exclue ; elle nous semble cependant peu probable.

Les examens de routine ont montré que dans la région lyonnaise, et notamment en Dombes, le mulot et divers campagnols sont souvent porteurs de Strongyloides ratti, tandis que le lérot ne l'est pas. Cette constatation peut s'expliquer parce que les Rongeurs cités en premier, qui sont fouisseurs, sont très en contact avec la terre ; ce n'est pas le cas du dernier, qui mène une vie arboricole à distance du sol. Les présents essais indiquent que la résistance d'Eliomys quercinus à ce parasitisme représente aussi une cause non négligeable de cette modalité écologique.

\section{Bibliographie}

ARREZA-GuZman (A.), 1937. - Recherches expérimentales sur le traitement de la strongyloïdose murine. Ann. Parasitol., 15, $\mathrm{n}^{\circ}$ 2, p. 125-145.

Brackett (S.) et Bliznick (A.), 1949. - An attempt to adapt Strongyloides ratti to the mouse. Journ. Parasitol., 35, $\mathrm{n}^{\circ} 1$, p. 41-44.

Langeron (M.), 1934. - Précis de Microscopie, $5^{\circ}$ édit., Paris, Masson édit.

Roman (E.), 1951. - Etude écologique et morphologique sur les Acanthocéphales et les Nématodes parasites des rats de la région lyonnaise. Mém. Mus. Hist. nat., nlle Série, A, Zool., 2, p. 49-270.

—, 1956. - Spécificité parasitaire de Strongyloides ratti du surmulot; effets de la cortisone sur l'infestation d'autres Rongeurs par ce Nématode. Ann. Parasitol., 31, $\mathrm{n}^{\circ}$ 5-6, p. $552-571$.

—, 1957. - Modification du procédé de Telemann pour la concentration des éléments parasitaires dans les selles riches en débris cellulosiques. Bull. Assoc. Diplômés Microbiol. Nancy, $\mathrm{n}^{\circ} 68$, p. $15-19$. 
—, 1959. - Sur la résistance à la réinfestation des rats parasités par le Nématode Strongyloides ratti. C.R. Soc. Biol., 153, n 11, p. 1795-1798.

—, 1964. - Observations sur les Strongyloides parasites des rats des champs. Bull. Assoc. Diplômés Microbiol. Nancy, $\mathrm{n}^{\circ}$ 96, p. 18-22.

—, 1964. - Etude biologique d'une souche de Strongyloides ratti provenant d'un rat des champs. Proceed. first internat. Congr. Parasitol., Roma, 1, p. 468-469, Pergamon Press, 1966.

SHELdON (A. J.), 1937. - Studies on active acquired resistance natural and artificial in the rat with Strongyloides ratti. Am. Journ. Hyg., 25, p. 53-65.

WertheIM (G.), 1959. - The infectivity of Strongyloides ratti for various Rodents hosts. Journ. Parasitol., 45, $\mathrm{n}^{\circ}$ 4, sect. 2, Abstract 67, p. 37. 